\title{
Vegetation Coverage Monitoring in Mu-us Sandy Land Based on Multiscale Remote Sensing Data-A Case Study of Yanchi County, Ningxia
}

\author{
Zhen Bian ${ }^{1}$, Wanxue You², Dian Yu ${ }^{2}$, Kebin Zhang ${ }^{3}$, Limeng Meng ${ }^{3}$, \\ Jin Chang 4
}

\author{
${ }^{1}$ School of Resources and Environment, University of Jinan, Jinan 250022, China; \\ ${ }^{2}$ Ningxia Habahu National Nature Reserve Administration, Wuzhong 751500, China; \\ ${ }^{3}$ Key Lab. of Soil \& Water Conservation and Desertification Combating, Ministry of Education, School \\ of Soil and Water Conservation, Beijing Forestry University, Beijing 100083, China;
}

${ }^{4}$ China Water Resources Pearl River Planning Surveying \& Designing Co., Ltd., Guangzhou 510610, China.

mushizh@163.com

Keywords: Digital image, Multiscale remote sensing data, Vegetation coverage, and Dynamic monitor.

\begin{abstract}
Due to the sparse and irregular distribution of vegetation in desertification area, the Low inversion precision of vegetation coverage and its change using single satellite remote sensing data become the bottleneck of further exploration of ecological evolution in this region. In order to improve the retrieval accuracy of vegetation coverage in desertification area, this article inverses and dynamic monitors the vegetation coverage of $\mathrm{Mu}$ Us Sandy Land in north of Yanchi County combining multi-source data include digital image, Landsat TM and MODIS-NDVI using scale conversion and two pixel model. The results showed that: (1) Vegetation information based on NDVI DC $_{\text {(normalized }}$ difference vegetation index based on digital camera) was accurately extracted with the classification accuracy up to $94.3 \%$, which provided a convenient and accurate method for ground survey and remote sensing revise of the vegetation coverage. (2) Vegetation coverage significantly increased after grain to green program and grazing prohibition measures in Yanchi county since the beginning of 2000-2002. (3) The method of vegetation coverage inversion based on multi-source remote sensing information provides a new reference for rapid and efficient vegetation monitoring in desertification areas.
\end{abstract}

\section{Introduction}

Vegetation coverage is one of key indicators for desertification monitoring in China arid and semi-arid regions [1]. The ground investigation of vegetation coverage is characterized by low precision, long time, large investment and difficult acquisition of large scale and long time-series data [2]. The advantages of the remote sensing inversion method of vegetation coverage are fully demonstrated with the wide application of remote sensing and geographic information system technology. Concrete methods include empirical mode method, vegetation index method and the metacolysis model method [3]. Wherein, the empirical model method is suitable only for specific regions and periods since remote sensing data is affected by atmosphere, sensor and other factors [5]. Compared with the experience model method, the vegetation index method has more widespread promotion value, but the local estimation accuracy is lower due to non-vegetation ground object spectral interference [5]. Pixel decomposition model refers that vegetation index mode is further perfected [6]. The most commonly used method is that the pixel is decomposed into dimidiate pixel modelof two components-vegetation and soil [7]. In summary, multi-scale remote sensing data is integrated to calculate the single component pixel value required for dimidiate pixel model accurately, thereby effectively improving remote sensing estimation precision of vegetation coverage, and 
providing important data support for deeply exploring occurrence and development mechanism of vegetation degradation and desertification.

In the paper, the agriculture and animal husbandry ecoton in the north of Ningxia Yanchi County at Mu-us sandy land is adopted as an example. The ground monitoring precision of vegetation coverage is improved, high-precision ground coverage data is combined with satellite remote sensing data at different scales and dimidiate pixel model method for establishing the vegetation coverage data with long time-series, thereby providing reliable basic data for studying vegetation evolution law in desertification areas.

\section{Overview of Study Area}

The study area is located in central and north part of Yanchi County, Ningxia, and south edge of Mu-us sandy land (Fig.1), which covers an area of $4063 \mathrm{~km}^{2}$. The study area belongs to the temperate continental climate with annual average temperature of $8.1{ }^{\circ} \mathrm{C}$; annual precipitation is $250 \sim 350 \mathrm{~mm}$. The soil is mainly composed of sierozem, heilu soil and sandy soil with local distribution of solonchak and and Baijiang soil. Grasslands and sandy vegetation are most advantageous according to vegetation types in natural distribution, which are accompanied with bush fallow, meadows, and desert vegetation. Xerophytic and mesoxerophytes are main categories [8].

\section{Data and Methods}

\subsection{Layout of Sample Plot}

In the research, NDVI data calculated by TM image was utilized for determining the area with prominent changes of vegetation coverage gradients in order to obtain vegetation coverage ground data at different gradients. Therefore, a total of 85 sample plots of four site conditions of Haba Lake (wetlands), Siertan (natural wetlands), Liuyangpu (artificial cultivating grass) and Shabianzi (semi-fixed dunes) were selected (figure 1). It was required in each sample plot that the vegetation coverage in and around the sample plot should be more uniform [9]. GPS was utilized to record coordinates on four corners of the sample plots. Each sample plot size was set as $30 \mathrm{~m} \times 30 \mathrm{~m}$ in field investigation in order to accurately match the Landsat TM images.

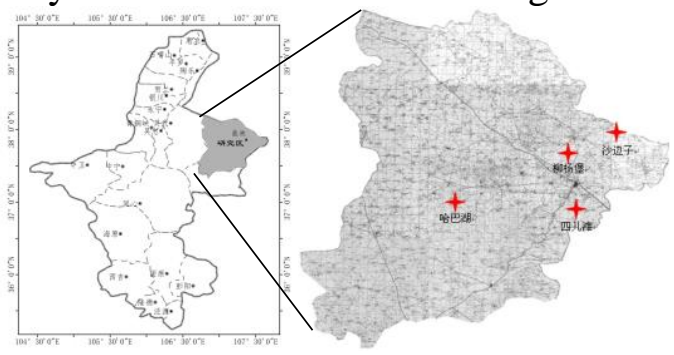

Fig. 1 The location of study area and distribution of sample plots

\section{Acquisition of ground vegetation coverage}

Field experiment was implemented in the study from July 30, 2010 to August 5, 2010. A digital camera was used for taking photos along diagonal line of the sample plot in a mode of being vertical to the ground.

\subsection{Data Preprocessing}

\subsubsection{Digital Image}

Photoshop software was utilized for combining the photos in each sample together.

\subsubsection{TM Data}

In the study, TM data on August 2 was selected, which was consistent with the ground investigation time. ENVI software was utilized for image radiation correction and FLAASH atmospheric correction. The geometrical correction error was controlled within 0.5 pixel.

\subsubsection{MODIS Data}

USGS MOD13A1 vegetation index product was used. Data with year range from 2000 to 2010 and time from the the $209^{\text {th }}$ day to the $224^{\text {th }}$ day each year was obtained, which was consistent with Landsat 
TM data. The precision of data products complied with the need of vegetation monitoring study at the scale [10].

\section{Experiment and Result}

\subsection{Calculation of Vegetation Coverage based on Digital Image}

In the study, the brightness values of the three channels (red)(green)(blue) of respective 100 pixels of plant and soil components in digital images were counted respectively. Wherein, the brightness value relationship of soil and vegetation in channel $\mathrm{G}$ and channel B was opposite (figure 2). Therefore, a normalized vegetation index based on digital camera $\left(N D V I_{D C}\right)$ was proposed.

$$
N D V I_{D C}=(G-R) /(G+R)
$$

In the formula, $N D V I_{D C}$ was a normalized vegetation index based on digital camera. $G$ and $R$ were respectively brightness values of red channel and green channel in the digital image. The vegetation pixel $N D V I_{D C}$ was higher than 0 , and the soil pixel $N D V I_{D C}$ was smaller than 0 . All pixel of the digital images were classified into vegetation and non-vegetation utilized $N D V I_{D C}$ in ArcGIS, and the vegetation coverage in different sample plots was counted.

The classification accuracy was between $94.3 \%$ and $99.8 \%$ based on 1000 pixels randomly selected in different vegetation communities, which was prominently higher than traditional ground vegetation investigation methods.
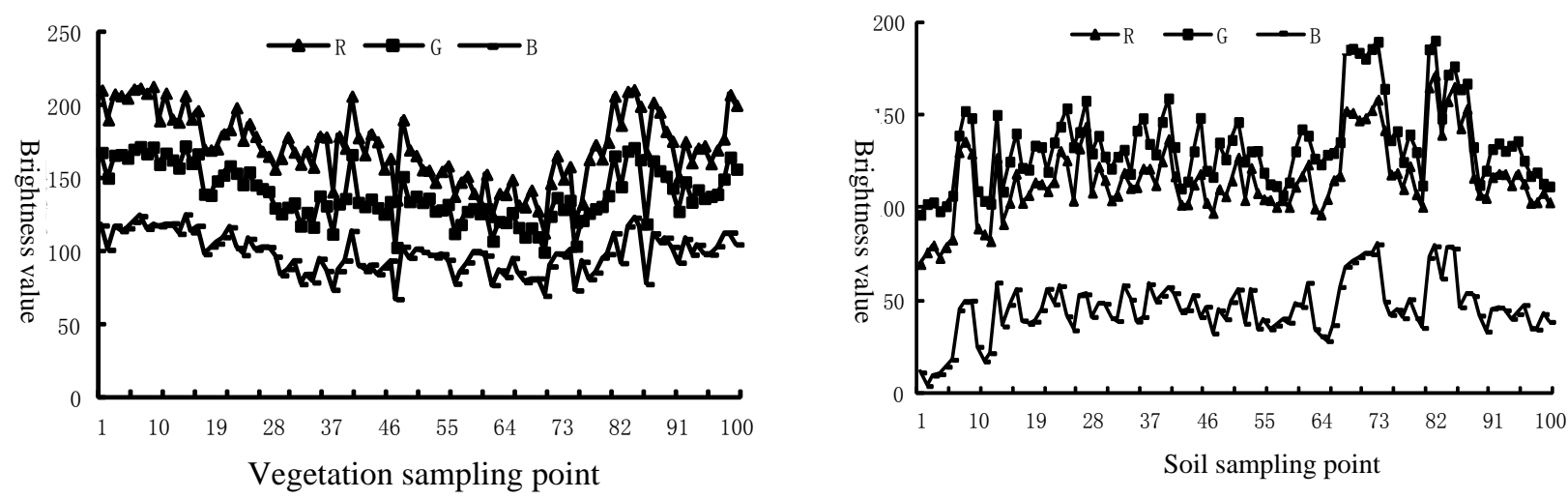

Fig.2 Vegetation and soil brightness value of R/G/B channel

\subsection{Inversion of Vegetation Coverage based on TM Vegetation Index}

Landsat TM data was utilized for calculating four vegetation indexes- RVI, NDVI, SAVI and MSAVI [11]. The results showed that NDVI had the largest correlation coefficient with vegetation coverage, and the mean square root error was the minimum (table 1).

Therefore, NDVI regression equation was utilized for inverting vegetation coverage in the study area, thereby obtaining a vegetation coverage classification diagram based on TM-NDVI (figure 3). Data of 10 remaining sample plots was used for testing the regression model.

Table 1 . The regression model of 4 vegetation indexes and vegetation coverage

\begin{tabular}{cccc}
\hline Vegetation index & Regression model & $\mathrm{R}^{2}$ & RMSE \\
\hline RVI & fvc $=0.2774$ rvi -0.2315 & 0.599 & 0.0774 \\
NDVI & fvc $=1.1615$ ndvi -0.0504 & 0.663 & 0.0709 \\
SAVI & fvc $=2.027$ savi -0.0841 & 0.642 & 0.0732 \\
MSAVI & fvc $=2.2151$ msavi -0.0727 & 0.619 & 0.0754 \\
\hline
\end{tabular}

Note: wherein fvc refers to the vegetation coverage calculated by vegetation index.

Figure 3 showed that the vegetation coverage in most study area was between $10 \%$ and $40 \%$. The vegetation coverage of county periphery farmland was more than $70 \%$. Forest coverage was between $50 \%$ and $70 \%$, the vegetation coverage in central sandy land was less than $20 \%$, and the inversion results were consistent with the field investigation situation. 


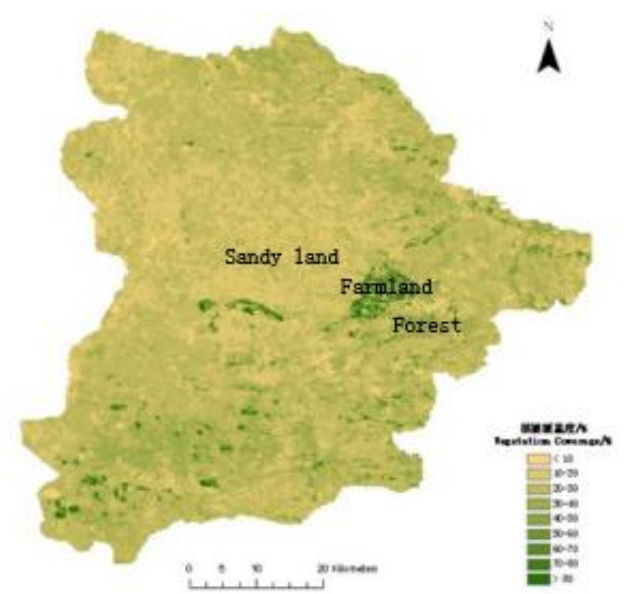

Fig.3 Vegetation coverage inversion based on TM-NDVI linear regulation model

\subsection{Vegetation Coverage Inversion based on MODIS-NDVI}

\subsubsection{Linear Regression Analysis of MODIS-NDVI and TM Estimation Vegetation Coverage}

The above TM vegetation coverage was resampled as data of $500 \mathrm{~m}$ resolution in order to explore the interannual change law of vegetation coverage in the study area, and the data was superimposed with MODIS NDVI data in the same period in 2010. 596 pixels were selected evenly. MODIS-NDVI value and TM vegetation coverage of each pixel were extracted. A linear regression model was established: $\mathrm{FVC}=1.302 \mathrm{NDVI}-0.099$. Wherein, FVC referred to TM vegetation coverage, and NDVI referred to the MODIS-NDVI value. The model passed $\alpha=0.05$ significance test, $\mathrm{R}^{2}=0.836$. The model and MODIS-NDVI data were utilized for inverting the vegetation coverage in early August 2010.

\subsubsection{Time Series Vegetation Coverage Inversion based on MODIS-NDVI}

Long time-series vegetation coverage was inverted on the basis of vegetation growth period MODIS-NDVI data from 2000 to 2010 , and the vegetation coverage changes in the study area were dynamically monitored.

It was set that the ground feature spectrum information of all pixels in MODIS image was weighed and composed of soil and vegetation information proportionally and linearly. $S_{v}$ and $S_{s}$ were respectively vegetation and soil spectral reflectivities in the pixel. $f_{c}$ was vegetation coverage. $S_{v e g}$ and $S_{\text {soil }}$ were respectively spectral reflectivity of pure vegetation pixel and pure soil pixel, namely:

$$
S=S_{v}+S_{s}=f_{c} \cdot S_{\text {veg }}+\left(1-f_{c}\right) \cdot S_{\text {soil }}
$$

The formula (2) was transformed. NDVIveg and NDVIsoil values represented NDVI values of pure vegetation and pure soil pixels, and the calculation formula for vegetation coverage was shown as follows:

$$
f_{c}=\frac{\mathrm{NDVI}-\mathrm{NDVI}_{\text {soil }}}{\mathrm{NDVI}_{\text {veg }}-\mathrm{NDVI}_{\text {soil }}}
$$

Therefore, the vegetation coverage was determined only by determining NDVI value $N D V I_{\text {soil }}$ and $N D V I_{v e g}$ of pure soil pixel and pure vegetation pixel. The linear regression model $\mathrm{FVC}=$ 1.3022NDVI - 0.0994 was utilized in order to calibrate $N D V I_{\text {veg }}$ and $N D V I_{\text {soil }}$ in the study area. FVC was set as 1 and 0 for respectively calculating $\mathrm{NDVI}_{\text {veg }}=0.8443$ and $\mathrm{NDVI}_{\text {soil }}=0.0763$. The theoretical value was used as $\mathrm{NDVI}_{v e g}$ and $\mathrm{NDVI}_{\text {soil }}$ values of each year for calculating the vegetation coverage in early August from 2000 to 2010 (figure 4). 

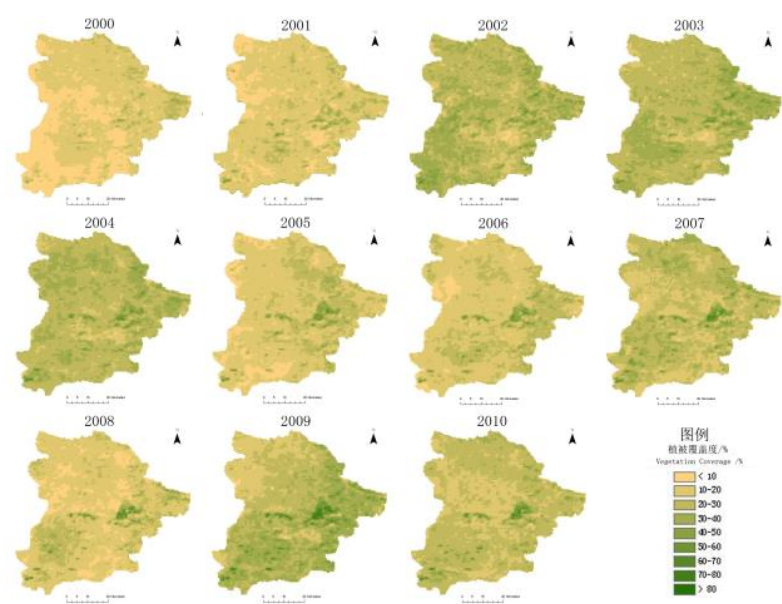

Fig. 4 Vegetation coverage in early August from 2000 to 2010

\subsection{Spatio-Temporal Change Analysis of Vegetation Coverage}

\subsubsection{Time Change Characteristics of Vegetation Coverage}

Figure 5 showed that the vegetation coverage was less than $20 \%$ as a whole in 2000 and 2001. There were continuous sandy lands in central and western regions. The vegetation coverage reached $40-50 \%$ as a whole from 2002 to 2004 . The inversion results year by year were consistent with the field investigation in previous years. The average vegetation coverage over the years was calculated in order to further analyze the spatial distribution law of vegetation coverage in the study area.

\subsubsection{Spatial Distribution Characteristics of Vegetation Coverage}

Average vegetation coverage data in the study area (figure 5) showed that the average vegetation coverage in the study area was mostly distributed in east and southwest regions. Most vegetation coverage in the east, central and southwest regions enjoyed good moisture condition, and beneficial conditions are provided for vegetation growth. The vegetation coverage in the northwest regions was less than $20 \%$, and the desertification was serious.

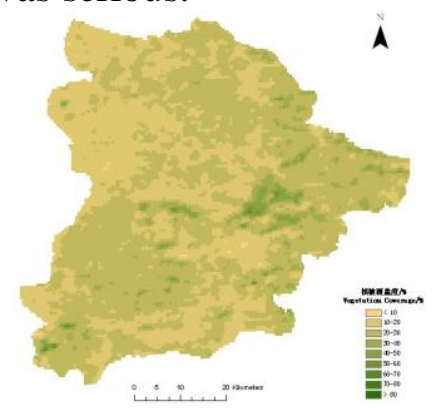

Fig.5 The average vegetation coverage (2000-2010)

\section{Conclusion}

In the paper, the vegetation coverage information at different scales is inverted on the basis of three remote sensing platforms of digital camera, Landsat TM and MODIS in order to solve the problems during traditional ground investigation and remote sensing inversion of vegetation coverage in desertification area-low precision and efficiency. The space-time change laws of vegetation coverage from 2000 to 2010 in the study area are analyzed. Main conclusions are shown as follows:

(1) Digital image R, G and B channel brightness values are utilized for establishing vegetation index based on visible light band, and the vegetation coverage in the sample plot is accurately estimated. The method is suitable for field precision gathering of vegetation coverage data in large scope.

(2) A vegetation coverage experience model and a dimidiate pixel model based on MODIS-NDVI is established through TM data linear regression model. Vegetation coverage data of time-series from 2000 to 2010 is inverted. The vegetation coverage is prominently increased after return of cultivated land into forest, fenced grassland and other measures were applied from 2001 to 2003. 
(3) Problems of traditional ground investigation method are avoided by the vegetation coverage inversion method based on multi-source remote sensing information, namely a lot of manpower and financial resources are wasted, and low basic data precision of traditional remote sensing inversion method. Timely and reliable data support can be provided for large-scale vegetation monitoring in desertification areas.

\section{References}

[1]. Li Xiaosong, Li Zengyuan, Gao Zhihai, et al. Estimation of Vegetation Cover in Desertified Regions from Hyperion Imageries Using NDVI and Partial Least Squares Regression. Journal of Desert Research. Vol. 31 (2011) No.01, p. 162-167.

[2]. Cao Yongxiang, Liu Xiaodan, Zhang Kebin, et al. Vegetation Coverage Change of Tsagan Us Oasis in Dulan County,Qinghai Province, China. Journal of Desert Research. Vol. 31(2011) No. 05, p. 1267-1272.

[3]. Qin Wei, Zhu Qingke, Zhang Xuexia, et al. Review of vegetation covering and its measuring and calculating method. Journal of Northwest Sci Tech University of Agriculture and Forestry (natural science edition). Vol. 34 (2006) No.09, p. 163-170.

[4]. Ding Yanling: Research on the Estimation of Fractional Vegetation Cover and the Validation of Fractional Vegetation Cover Product (Doctor degree, Graduate University of the Chinese Academy of Sciences (Northeast Institute of Geography and Agricultural Ecology), China 2015). p. 179.

[5]. Li Miaomiao: The method of vegetation fraction estimation by remote sensing (Master degree, Institute of Remote Sensing Applications Chinese Academy of Sciences, China 2003). p. 113.

[6]. [6]. Gu Zhujun, Zeng Zhiyuan. Overview of Researches on Vegetation Coverage in Remote Sensing. Research on Water and Soil Conservation. Vol. 12 (2005) No.02, p. 18-21.

[7]. Zribi M,Le Hegarat-Mascle S,Taconet O, et al. Derivation of wild vegetation cover density in semi-arid regions: ERS2/SAR evaluation. International Journal of Remote Sensing. Vol.24 (2003) No.6, p. 1335-1352.

[8]. Du Lingtong, Tian Qingjiu. Vegetation Coverage Variations in Ningxia during 1999--2009 and Its Relationships with Climatic Factors. Journal of Desert Research. Vol.32 (2012) No.05, p. 1479-1485.

[9]. Cui Yaoping, Wang Ranghui, Liu Tong, et al. Extraction of Vegetation Information in Arid Desert Area based on Spectral Mixture Analysis — - a Case in the Western Gurbantunggut Desert. Journal of Desert Research. Vol. 30 (2010) No.02, p. 334-341.

[10]. Chen Yanmei, Gao Jixi, Diao Zhaoyan, et al. Spectral models for estimating grassland vegetation coverage on Hulunbeier Grassland, Inner Mongolia, China. China Environmental Science. Vol. 30 (2010) No.09, p. 1287-1292.

[11]. Xia Zhaohua: The studies on dynamic of vegetation based on NDVI time series (Master degree, Beijing Forestry University, China 2007). p. 115. 